\title{
Autophagy eliminates ER membrane reorganization induced by Bcl-2 inhibitor in HeLa cells
}

\author{
XIAONING LI $^{1}$, YONGWEI KANG ${ }^{1}$, BINGXUAN JIAO ${ }^{2}$, MEIHUI XIA ${ }^{3}$, YAO WU ${ }^{1}$ and LIANKUN SUN ${ }^{1}$ \\ ${ }^{1}$ Department of Pathophysiology, Basic Medical College, Jilin University; ${ }^{2}$ School of Public Health, Jilin University; \\ ${ }^{3}$ Department of Obstetrics and Gynecology, The First Hospital, Jilin University, Changchun, Jilin 130021, P.R. China
}

Received March 22, 2016; Accepted August 4, 2016

DOI: 10.3892/or.2016.5209

\begin{abstract}
The endoplasmic reticulum (ER) is a membranous network within cells that is important for several cellular functions including translation and folding of secretory and membrane proteins, lipid biogenesis and sequestration of $\mathrm{Ca}^{2+}$. Disruption of ER structure might affect the normal physiology of the cells. In yeast, expansion of the ER is observed under unfolded protein response (UPR) and subsequently induces autophagy initiated from the ER. In this study, we demonstrated a drastic and specific ER membrane reorganization (EMR), characterized by the clustering of the ER membrane into large and compact aggregates and occurring independent of UPR in HeLa cells treated with S1 combined with ABT-737. Subsequently, combined with S1 and ABT-737 triggered autophagy. Herein, we report a key step for removal of damaged and superfluous cellular constituents, by a mechanistic link between ER aggregation and autophagic activation. Our study is the first time to show that autophagy may be a way to remove the ER membrane reorganization induced by Bcl-2 inhibitors ABT-737 and S1 and it may help us to analyze autophagy in certain diseases.
\end{abstract}

\section{Introduction}

Endoplasmic reticulum has multiple physiological functions, including involvement in the processes of protein synthesis, protein folding, post-translational modifications, protein transportation, and protein secretion (1-3). There are various network structures of endoplasmic reticulum, including tubular, paliform, and sheet structure $(3,4)$. Several studies have reported the use of the Connectivity Map database to identify over 20 kinds of small molecule compounds that induce reorganization of endoplasmic reticulum membrane,

Correspondence to: Dr Liankun Sun, Department of Pathophysiology, Basic Medical College, Jilin University, 126 Xinmin Street, Changchun, Jilin 130021, P.R. China

E-mail:sunlk@jlu.edu.cn

Key words: Bcl-2 inhibitor, ER membrane reorganization, unfolded protein response, autophagy indicating that endoplasmic reticulum undergoes different degrees of dynamic changes under stress stimulation. The reorganization of endoplasmic reticulum membrane is a general form of cellular response to stress (5). In an investigation by Varadarajan and colleagues, the endoplasmic reticulum of tumor cells treated with the pan-Bcl-2 inhibitor apogossypol underwent a dramatic and unique morphological change. This phenomenon, known as endoplasmic reticulum membrane shrinkage, is seen under an electron microscope as massive aggregation of the proteins in the endoplasmic reticulum membrane and was defined by Varadarajan and colleagues as the reorganization of endoplasmic reticulum membrane. Apogossypol induces a variety of different cell lines to generate the same morphological change in endoplasmic reticulum membrane; these include Jurkat $\mathrm{T}$ lymphocytes, HeLa cells, mouse embryonic fibroblasts (MEFs), Chinese hamster ovarian carcinoma cells (CHOs), Saccharomycetes, and Schizosaccharomyces pombe (5). This shows that the reorganization of endoplasmic reticulum membrane is conservative in nature, and maybe a new form of cellular stress response.

Since this stress response shows conservatism and universality, it may be closely related to the mechanism of cellular damage and anti-damage. Explanation of this mechanism may provide further insight into the toxicity and side effects of drugs, and the mechanism of action of antitumor drugs. Because this dynamic change precedes the unfolded protein response (UPR) of endoplasmic reticulum, the relationship of the reorganization of endoplasmic reticulum membrane and the UPR is very interesting.

It is widely accepted that Bcl-2 family proteins are located in the endoplasmic reticulum. Combined with the aforementioned results of pan-Bcl-2 inhibitor studies, some researchers have speculated that Bcl-2 family proteins may have the function of regulating morphology of endoplasmic reticulum and may be involved in the reorganization of endoplasmic reticulum membrane. Some researchers have reported that Bcl-XL induces endoplasmic reticulum lumen swelling through interacting with BAK, leading to cell death in $293 \mathrm{~T}$ cells co-expressing Bcl-XL and BAK (6). In addition, the Bcl-2 family protein member BH3-only protein, BNIP, has an effect on the morphology of endoplasmic reticulum by connecting with adhesion protein receptor syntaxin-18, and is located in endoplasmic reticulum (7). Although this investigation provides evidence that $\mathrm{Bcl}-2$ family proteins affect the 
morphological structure of endoplasmic reticulum, strong evidence of morphology and function is lacking.

In our previous study, we found that the Bcl-2 small molecular inhibitor S1 was different from other BH3-only mimetics, and can inhibit high expression of the anti-apoptotic protein Mcl-1 in various tumors. Both S1 and ABT-737 induced autophagy through interfering with the interaction of Bcl-2 and Beclin-1 (8,9). In our follow-up study we found that both $\mathrm{S} 1$ and ABT-737 induced slight reorganization of endoplasmic reticulum membrane. Furthermore, S1 combined with ABT-737 induced severe reorganization of endoplasmic reticulum membrane. Thus, we speculate that $\mathrm{Bcl}-2$ inhibitors may cause autophagy through inducing the reorganization of endoplasmic reticulum membrane. This suggests that autophagy was induced by Bcl-2 inhibitors and may be related to the elimination of damaged organelles. We found that endoplasmic reticulum partly disappeared in the presence of persistent membrane reorganization stress. It has yet to be determined whether reorganization of endoplasmic reticulum membrane is a self-healing function of endoplasmic reticulum, or the endoplasmic reticulum portion of morphology change occurs through elimination, and if it is through a clearing process, how it is removed (10).

There is evidence that autophagy is responsible for eliminating long-life proteins, protein aggregation, or damaged organelles such as endoplasmic reticulum and mitochondria. For example, in adult myocardial cells with high expression of BNIP3, the degradation of mitochondria increased by autophagy (11). After knocking down $\alpha$ SNAP in human epithelial cells the function of Golgi apparatus is lost, and the Golgi apparatus with loss of function is eliminated by autophagy (12). Therefore, exploring the relationship of autophagy and the reconstruction of endoplasmic reticulum membrane may identify the mechanism of the reorganization of endoplasmic reticulum membrane.

In this study, we used Bap31 and calnexin as markers of endoplasmic reticulum membrane, this method has been widely used and marks the endoplasmic reticulum membrane location. Using RNAi technology and higher special inhibitor, we explored the relationship of endoplasmic reticulum and unfolded protein response, to assess whether autophagy is involved in morphological changes of endoplasmic reticulum part removal. This is important for determining the function of autophagy under new cellular stress forms, at the same time our study also provides important evidence for the involvement of autophagy in regulating organelle morphology.

\section{Materials and methods}

Reagents and antibodies. Fetal bovine serum (FBS) and Roswell Park Memorial Institute IMDM culture medium were purchased from Invitrogen. 3-Methyladenine (3-MA) and Chloroquine (CQ) were purchased from Sigma. The BH3 mimetic S1 was supplied by Professor Zhichao Zhang and dissolved in dimethyl sulfoxide (DMSO). FITC/Texas Red-conjugated secondary antibodies were purchased from Santa Cruz Biotechnology Inc. (Santa Cruz, CA, USA). Enhanced chemiluminescence (ECL) reagents were purchased from Thermo Scientific. Antibodies anti-Bap31, anti-calnexin, anti-CHOP, anti-Bip, anti-eIF2a, anti-p-eIF2a, anti-LC3, anti-Beclin-1 and anti-Atg12 were purchased from Santa Cruz Biotechnology Inc., and horseradish peroxidase-conjugated anti-mouse and anti-rabbit immunoglobulins were purchased from Proteintech (Chicago, IL, USA).

Cell culture. Human cervical cancer HeLa cells were cultured at $37^{\circ} \mathrm{C}$ under $5 \% \mathrm{CO}_{2}$ in IMDM culture medium supplemented with $10 \%$ FBS. The cultures were passaged by $0.4 \%$ trypsinization, and fresh medium was changed for 2 days.

RNA interference. Bcl-2 and Mcl-1 shRNA plasmids were purchased from Shanghai GenePharma Co., Ltd. (Shanghai, China). The sh-RNA plasmid insert sequence was used as follows: sh-Bcl-2-1072: CCGGGAGATAGTGATGAAGTA; sh-Bcl-2-2659: CGCCCTGTGGATGACTGAGTA; sh-Mcl-11252: GCACACCTGGATCCAGGATAA; sh-Mcl-1-3153: CCGCATTTAATTCATGGTATT; Scr: CCTGTGGAACG TGTCACGCTT.

For transformation using DH5 $\alpha$ Escherichia coli competent cells, the competent cells and the transformation protocol were prepared according to a modified procedure based on that of Zhang and colleagues (13).

Stable transfected cell lines were developed using sh-RNA plasmids, which contain a neomycin resistance marker for the selection of stably transfected cells. HeLa cells were transfected with the sh-Bcl-2-1072/2659, sh-Mcl-1-1252/3153 and Scr in a 6-well plate with Lipofectamine 2000 transfection reagent. All procedures were performed according to the reagent supplier's guidance. A selective medium was added containing G418 $(600 \mu \mathrm{g} / \mathrm{ml})$ as the selective antibiotic pressure. Every 2-3 days, the medium was replaced with fresh medium. The selection was continued until all of the nontransfected cells had died. The surviving cells were then split into a lower density in a 12 -well plate. After transfection, cells were cultured for $48 \mathrm{~h}$ before being harvested or treated as indicated.

Western blot analysis. Cells were washed with phosphatebuffered saline (PBS) twice and harvested by scraping into $300 \mu \mathrm{l}$ of RIPA lysis buffer. Total cell lysates were lysed for $15 \mathrm{~min}$ on ice, and at $4^{\circ} \mathrm{C}$ for $45 \mathrm{~min}$, and after centrifugation at $12,000 \mathrm{x} \mathrm{g}$ for $10 \mathrm{~min}$ at $4^{\circ} \mathrm{C}$, the supernatant was collected. Protein concentrations in the supernatants were determined by the Bio-Rad reagent (Hercules, CA, USA). Equal amounts of proteins $(30 \mu \mathrm{g})$ were subjected to sodium dodecyl sulfate-polyacrylamide gel electrophoresis (SDS-PAGE) and transferred onto PVDF membrane (Millipore, Billerica, MA, USA). Transfer efficiency was checked with Ponceau staining. The blots were blocked in Tris-buffered saline containing nonfat dry milk $5 \%(\mathrm{w} / \mathrm{v})$, probed with specific primary antibodies overnight at $4^{\circ} \mathrm{C}$. After washing with PBS containing $0.05 \%$ Tween-20 (PBST), the membrane was incubated with a peroxidase-conjugated secondary antibody for $2 \mathrm{~h}$ at room temperature. Finally, each membrane was probed to detect $\beta$-actin. The final dilutions and incubation times suggested by the manufacturer were used for each antibody. Immunodetection was performed using the ECL solution and images captured by Syngene Bio Imaging (Synoptics, Cambridge, UK). Densitometry quantitation of the bands was also performed using equipment from Syngene Bio Imaging. 
A
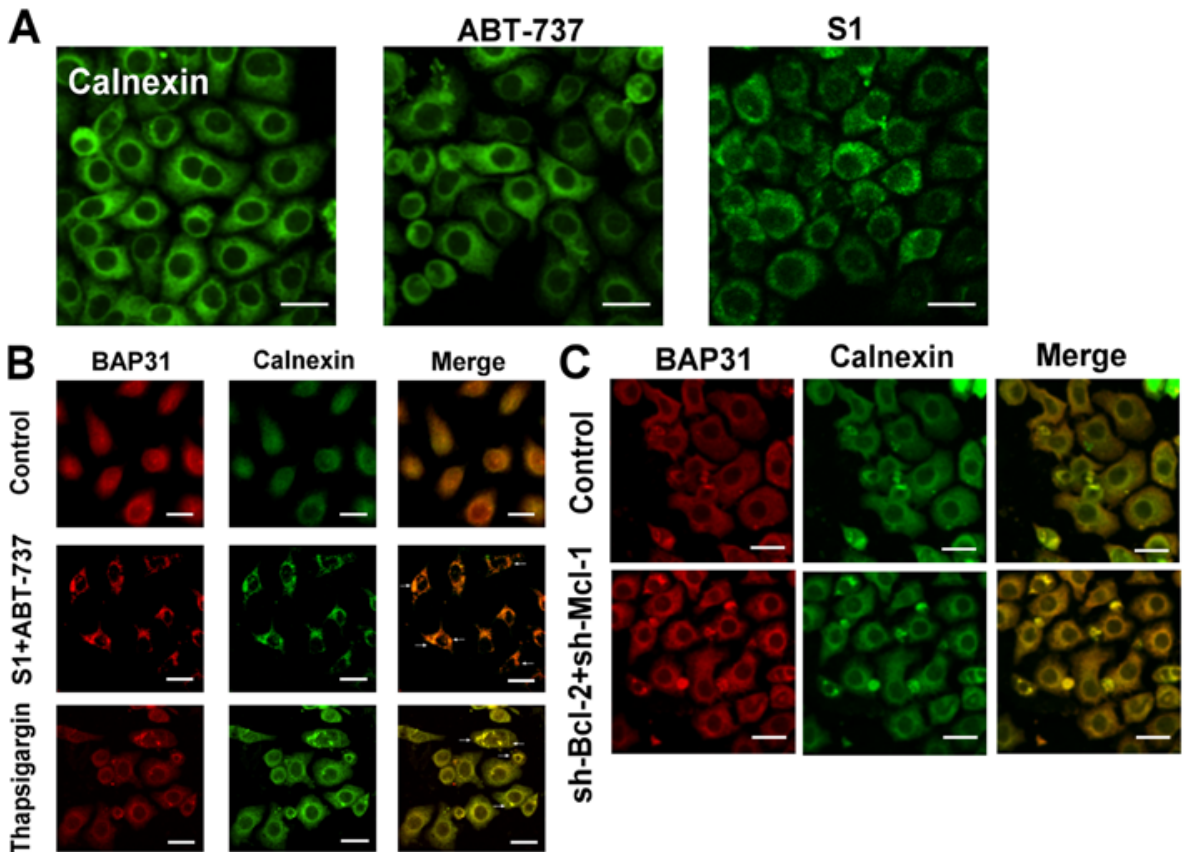

$\frac{\dot{c}}{\text { b }}$
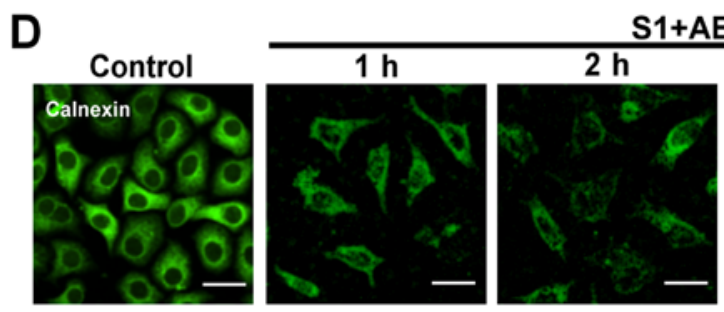

S1+ABT-737
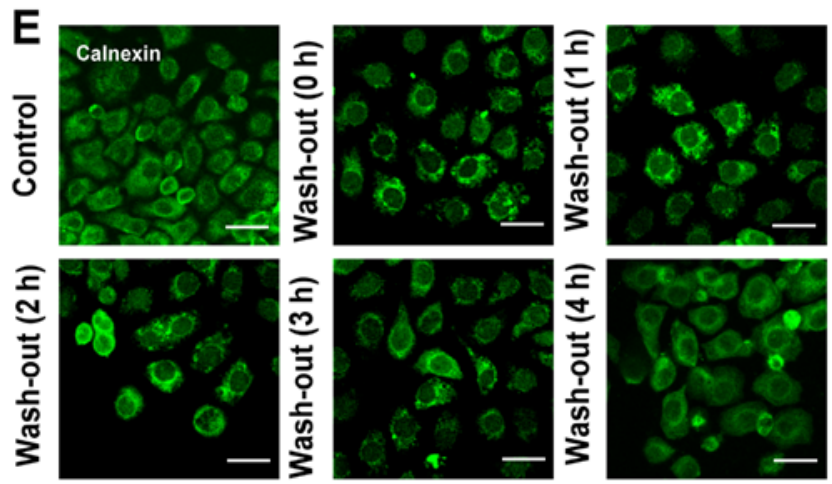

Figure 1. S1 combined with ABT-737 induces ER membrane remodeling in HeLa cells. (A) HeLa cells exposed for $8 \mathrm{~h}$ to $10 \mu \mathrm{mol} / 1 \mathrm{ABT}-737 \mathrm{or} 8 \mu \mathrm{mol} / 1 \mathrm{~S} 1$, and subsequently immunostained for ER membrane protein (calnexin) exhibited aggregation of the ER membrane proteins (scale bar, $20 \mu \mathrm{m}$ ). (B) HeLa cells exposed for $4 \mathrm{~h}$ to $15 \mu \mathrm{mol} / 1 \mathrm{ABT}-737$ and $8 \mu \mathrm{mol} / 1 \mathrm{~S} 1$ and immunostained for ER membranes (Bap31 and calnexin) exhibited clustering of the ER membrane proteins, similar to those following exposure to thapsigargin (5 $\mu \mathrm{mol} / 1)$ (scale bar, $20 \mu \mathrm{m}$ ). (C) Downregulation of Bcl-2 and Mcl-1, resulted in the formation of ER membrane aggregation (Bap31 and calnexin). (D) Calnexin-containing ER membrane aggregates undergo time-dependent formation and coalescence in HeLa cells exposed to $15 \mu \mathrm{mol} / 1$ ABT-737 and $8 \mu \mathrm{mol} / 1$ S1 (scale bar, $20 \mu \mathrm{m}$ ). The ER membrane aggregates were visible within $1 \mathrm{~h}$. (E) The Bap31-containing ER membrane aggregates, formed in HeLa cells following exposure for $4 \mathrm{~h}$ to $15 \mu \mathrm{mol} / 1$ ABT-737 and $8 \mu \mathrm{mol} / 1 \mathrm{~S} 1$, dispersed rapidly after wash out (scale bar $20 \mu \mathrm{m})$.

Immunofluorescence staining and confocal laser microscopy. Cells were cultured onto coverslips in 24-well plates overnight, treated with $8 \mu \mathrm{mol} / 1 \mathrm{~S} 1$ and $15 \mu \mathrm{mol} / 1 \mathrm{ABT}-737$ for different times, and fixed with $4 \%$ paraformaldehyde for $30 \mathrm{~min}$. After permeabilization with $0.1 \%$ Triton X-100 for $5 \mathrm{~min}$, followed by washing 3 times with PBS, the cells were sealed with bovine serum albumin for $1 \mathrm{~h}$ and incubated with a primary antibody against Bap31, calnexin, Atg12, Beclin-1 or LC-3 (1:100 dilution) overnight at $4^{\circ} \mathrm{C}$. The cells were rinsed and incubated with FITC/Texas Red-conjugated secondary antibodies (1:400 dilution; Santa Cruz Biotechnology Inc.) for $1 \mathrm{~h}$ at room temperature, washed with PBS three times, and examined using the Olympus FV1000 confocal laser microscope. Line profiles were carried out using Image Tool software (Image Pro Plus6.0) to quantify the quality of immunofluorescence for some images. The line profile command displays a twodimensional graph that represents the intensities of pixels along a line within an image. This provides a graphical representation of background and immunofluorescence signal.

Statistical analysis. Results are expressed as the mean \pm standard deviation (SD) of repeated experiments, as indicated in 
A

Bcl-2
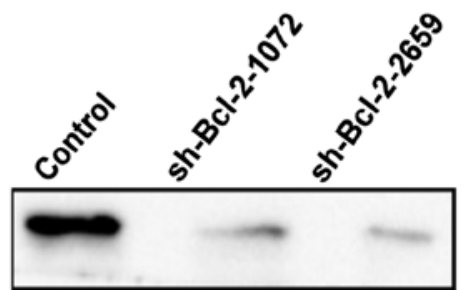

actin

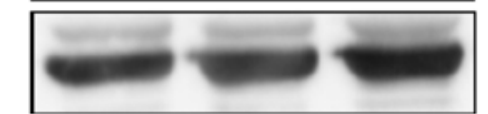

B

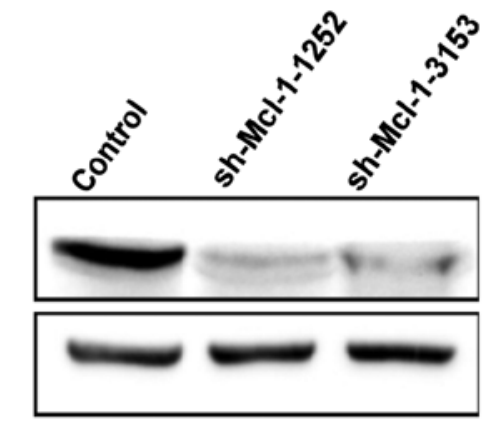

C

$\mathrm{Bcl}-2$

Mcl-1

actin
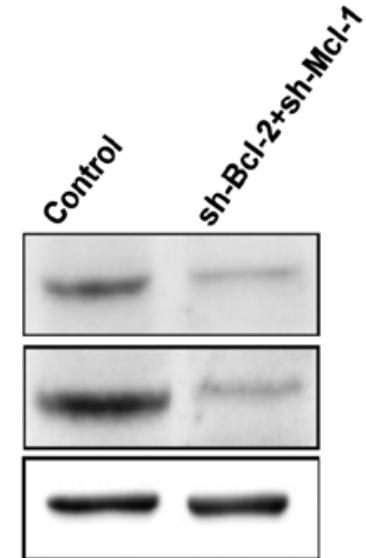

Figure 2. The knockdown efficiency of Bcl-2 and Mcl-1 with small interfering RNA in HeLa cells. (A) Western blot analysis for the expression of Bcl-2 in HeLa cells were transfected with sh-Bcl-2-1072/2659. (B) Western blot analysis for the expression of Mcl-1 in HeLa cells were transfected with sh-Mcl-1-1252/3153. (C) Western blot analysis for the expression of Bcl-2 and Mcl-1 in HeLa cells were transfected with sh-Bcl-2-2659 and sh-Mcl-1-3153.

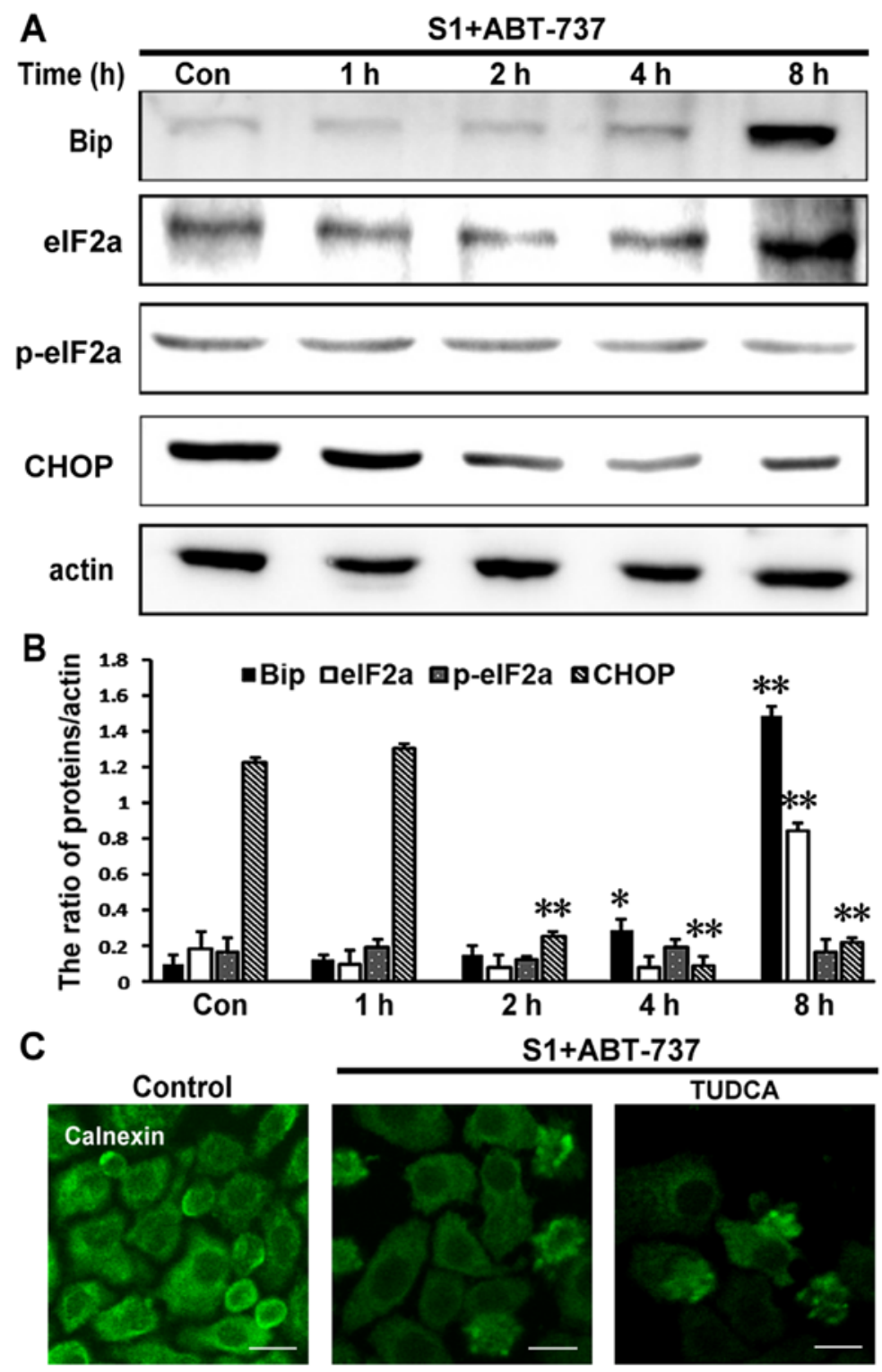

Figure 3. The reorganization of endoplasmic reticulum membrane earlier than the changes of the expression of UPR-related proteins. (A) Western blot analysis for the expression of Bip, eIF2a, p-eIF2a and CHOP in HeLa cells treated with $15 \mu \mathrm{mol} / 1 \mathrm{ABT}-737$ and $8 \mu \mathrm{mol} / 1 \mathrm{~S} 1$ for various times $(0,1,2,4,8 \mathrm{~h})$. (B) Quantitative analysis of Bip, eIF2a, p-eIF2a and CHOP protein levels from (A). Data are presented as mean \pm SD, $n=3,{ }^{*} \mathrm{P}<0.05,{ }^{* * *} \mathrm{P}<0.01$ versus control group. (C) Confocal microscopy of calnexin in the cytoplasm of HeLa cells treated with $15 \mu \mathrm{mol} / 1 \mathrm{ABT}-737$ and $8 \mu \mathrm{mol} / 1 \mathrm{~S} 1$ or combined with $500 \mu \mathrm{mol} / 1$ TUDCA in HeLa cells for $4 \mathrm{~h}$ (scale bar, $20 \mu \mathrm{m}$ ). 
A

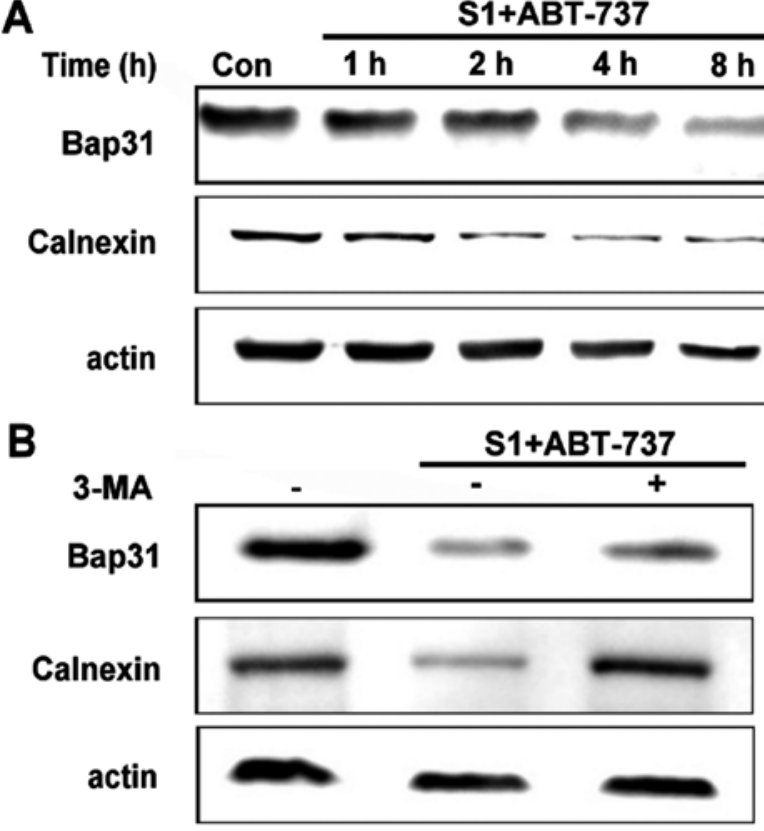

C

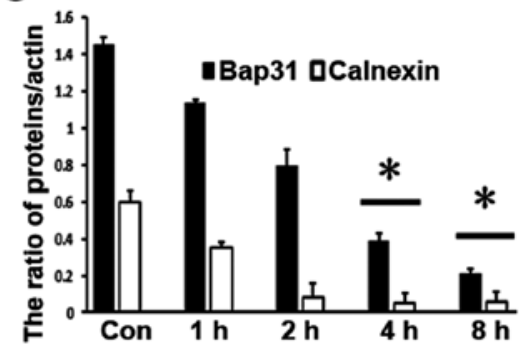

D

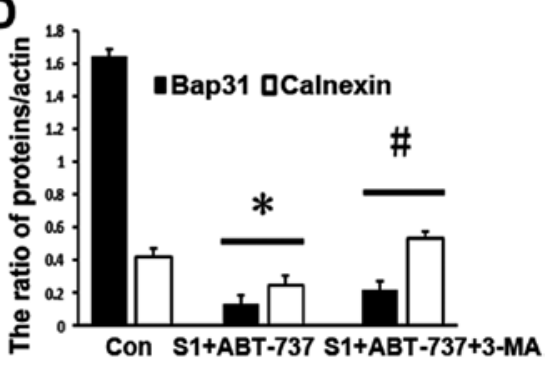

Figure 4. Degradation of ER membrane proteins is blocked by 3-MA. (A) Western blot analysis for the expression of Bap31, calnexin in HeLa cells treated with $15 \mu \mathrm{mol} / 1 \mathrm{ABT}-737$ and $8 \mu \mathrm{mol} / 1 \mathrm{~S} 1$ for various times $(0,1,2,4,8 \mathrm{~h})$. (B) Western blot analysis for the expression of Bap31, calnexin in HeLa cells treated with $15 \mu \mathrm{mol} / 1 \mathrm{ABT}-737$ and $8 \mu \mathrm{mol} / 1 \mathrm{~S} 1$ or combined with $10 \mathrm{mM} 3-\mathrm{MA}$ in HeLa cells for $4 \mathrm{~h}$. (C) Quantitative analysis of Bap31, calnexin protein levels from (A). Data are presented as mean $\pm \mathrm{SD}, \mathrm{n}=3,{ }^{*} \mathrm{P}<0.05$ versus control group. (D) Quantitative analysis of Bap31, calnexin protein levels from (B). Data are presented as mean $\pm \mathrm{SD}, \mathrm{n}=3,{ }^{*} \mathrm{P}<0.05$ versus control group, ${ }^{\#} \mathrm{P}<0.05$ versus $\mathrm{S} 1$ combined with $\mathrm{ABT}-737$ group.

the figure legends. Data are representative of three independent experiments performed in triplicate. Statistical differences were evaluated using the paired two-tailed Student's t-test. Differences were considered statistically significant for values of $\mathrm{P}<0.05$.

\section{Results}

S1 combined with ABT-737 induces ER membrane remodeling in HeLa cells. Although initially identified as central regulators of apoptosis at the mitochondrial level, the importance of Bcl-2 proteins at the endoplasmic reticulum is now well established (14). In our study, we used fluorescence microscopy to monitor endoplasmic reticulum membrane aggregation (5), and found that either $10 \mu \mathrm{mol} / 1$ ABT-737, which inhibits Bcl-2 but not Mcl-1, or $8 \mu \mathrm{mol} / 1 \mathrm{~S} 1$, which inhibits Mcl-1, induced a lower rate of endoplasmic reticulum reorganization after $8 \mathrm{~h}$ treatment (Fig. 1A). However, when the two inhibitors were applied together, the endoplasmic reticulum membrane reorganization was increased at $4 \mathrm{~h}$. A clustering of endoplasmic reticulum membrane proteins (Bap31/calnexin) was observed in cells treated with $8 \mu \mathrm{mol} / 1$ S1 combined with $15 \mu \mathrm{mol} / 1$ ABT-737 (Fig. 1B). When applied together, S1 and ABT-737 induced a profound aggregation of membranous structures resembling those induced by $5 \mu \mathrm{mol} / 1$ thapsigargin (Tg) (Fig. 1B). These results suggested that Bcl-2 family proteins might play an important role in endoplasmic reticulum membrane reorganization.

We also observed endoplasmic reticulum membrane reorganization in HeLa cells which knock down Bcl-2 and Mcl-1 with small interfering RNA (Figs. 1C and 2). In addition, calnexin clusters were seen at $1 \mathrm{~h}$ in HeLa cells treated with S1 combined with ABT-737, gradually becoming more serious with prolonged exposure time (Fig. 1D). Rapid and complete dispersal of endoplasmic reticulum membrane reorganization was observed when S1 and ABT-737 were washed out (Fig. 1E), indicating the reorganization of the endoplasmic reticulum membranes is reversible.

S1 combined with ABT-737 induces endoplasmic reticulum membrane remodeling earlier than UPR-related changes in HeLa cells. We investigated whether the inhibition of Bcl-2 and Mcl-1 by $8 \mu \mathrm{mol} / 1 \mathrm{~S} 1$ and $15 \mu \mathrm{mol} / \mathrm{l}$ ABT-737 could trigger canonical endoplasmic reticulum stress. Results showed that expression of Bip, eIF2a, and CHOP was upregulated following treatment with S1 combined with ABT-737 in HeLa cells, however, S1 combined with ABT-737 in HeLa cells had little effect on the expression of p-eIF2a. This results showed that the UPR-related changes were detected after $8 \mathrm{~h}$, whereas the reorganization of the endoplasmic reticulum membranes occurred before $8 \mathrm{~h}$ (Fig. $3 \mathrm{~A}$ and $\mathrm{B},{ }^{*} \mathrm{P}<0.05,{ }^{* *} \mathrm{P}<0.01$ ). Furthermore, $500 \mu \mathrm{mol} / 1$ tauroursodeoxycholate (TUDCA), an endoplasmic reticulum stress inhibitor, failed to abolish endoplasmic reticulum membrane reorganization induced by S1 combined with ABT-737 in HeLa cells (Fig. 3C).

S1 combined with ABT-737 downregulates endoplasmic reticulum membrane proteins. Of note, in this study we found that S1 combined with ABT-737 markedly decreased the expression of the endoplasmic reticulum proteins calnexin and Bap31 as early as $4 \mathrm{~h}$ (Fig. $4 \mathrm{~A}$ and $\left.\mathrm{C},{ }^{*} \mathrm{P}<0.05\right)$. Protein degradation is a fundamental cellular process that is executed by the separately regulated autophagy-lysosomal and ubiquitin-proteasome systems (15). As expected, degradation of ER membrane proteins was blocked by $10 \mathrm{mM} 3-\mathrm{MA}$ (Fig. 4B and $\mathrm{D},{ }^{*} \mathrm{P}<0.05,{ }^{\#} \mathrm{P}<0.05$ ). These results suggested that 
A
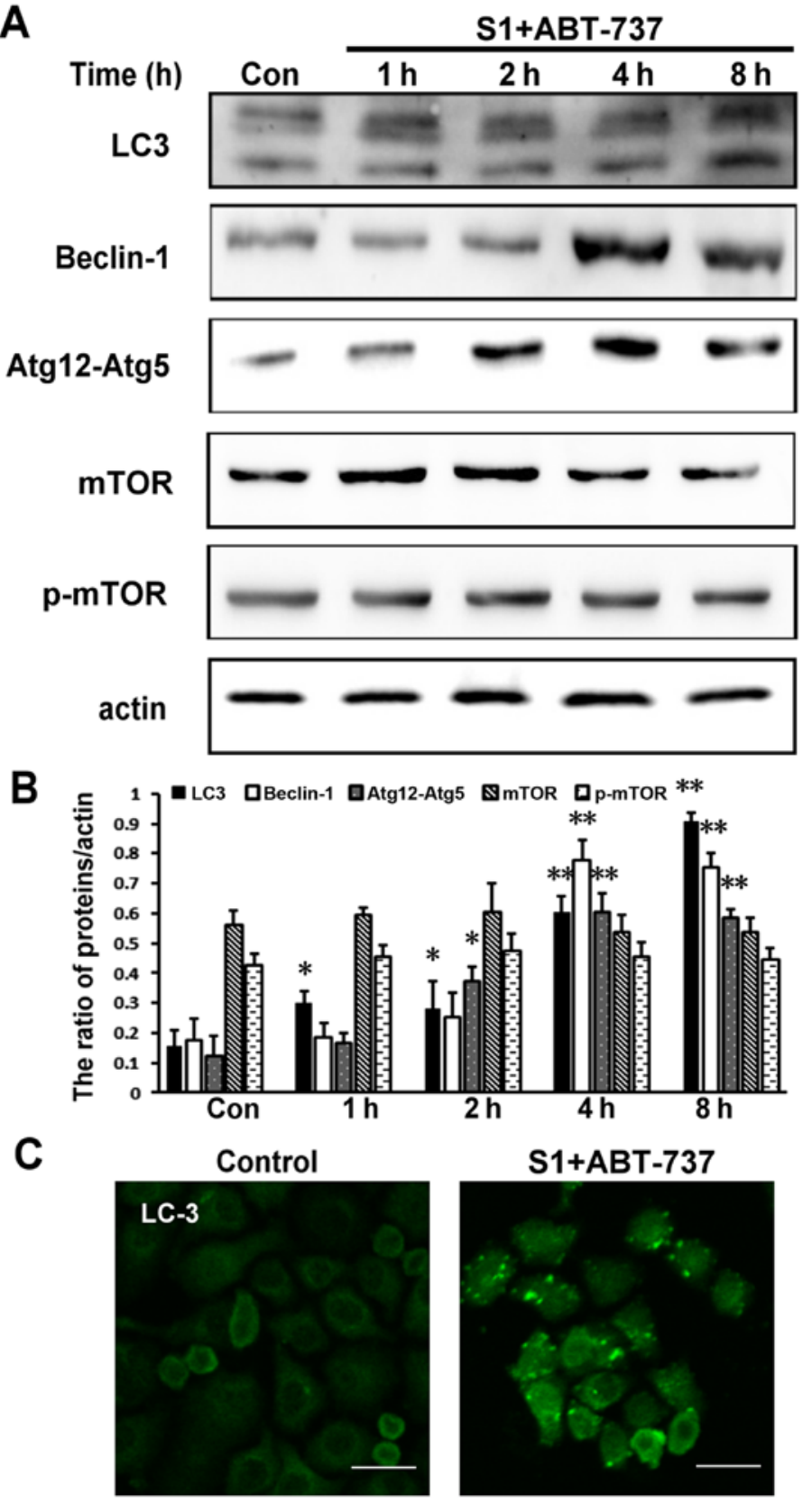

Figure 5. S1 combined with ABT-737 induces autophagy in HeLa cells. (A) Western blot analysis for the expression of LC3, Beclin-1, Atg12-Atg5, mTOR and p-mTOR in HeLa cells treated with $15 \mu \mathrm{mol} / 1$ ABT-737 and $8 \mu \mathrm{mol} / 1 \mathrm{~S} 1$ for various times $(0,1,2,4,8 \mathrm{~h})$. (B) Quantitative analysis of LC3, Beclin-1, Atg12-Atg5, mTOR and p-mTOR protein levels from (A). Data are presented as mean $\pm \mathrm{SD}, \mathrm{n}=3,{ }^{*} \mathrm{P}<0.05,{ }^{* *} \mathrm{P}<0.01$ versus control group. (C) Confocal microscopy of LC3 in the cytoplasm of HeLa cells treated with $15 \mu \mathrm{mol} / 1$ ABT-737 and $8 \mu \mathrm{mol} / 1 \mathrm{~S} 1$ for the $8 \mathrm{~h}$.

autophagy might be associated with decrease of endoplasmic reticulum membrane proteins.

S1 combined with ABT-737 induces autophagy in HeLa cells. In addition to regulating apoptosis, it has been reported that Bcl-2 protein has a function in autophagy (16). Moreover, research has shown that autophagy plays an important role in cellular quality control and is responsible for removing protein aggregates and dysfunctional organelles (17). In addition, our previous study reported that $\mathrm{S} 1$ induced autophagy through inhibiting the interaction of Bcl-2 and Beclin1, and then inducing the release of autophagy initiation protein Beclin1, finally inducing autophagy in U251 cells (8) and the mechanism of ABT-737 induces autophagy similarly to S1 (9).
Therefore, we further explored whether S1 combined with ABT-737 could indeed induce autophagy in HeLa. Atg12-Atg5 and Atg8 (LC3), the two ubiquitin-like conjugation systems, are required for the initiation and expansion of autophagosomal membranes (17).

Our results showed that the expression of the Atg12-Atg5 complex was increased in HeLa cells treated with S1 combined with ABT-737 (Fig. 5A and B, ${ }^{*} \mathrm{P}<0.05,{ }^{* *} \mathrm{P}<0.01$ ). When autophagy occurs, LC3 protein appears as dots, and the soluble form of LC3 (LC3-I) changes into the lipidated and autophagosome-associated form (LC3-II) $(18,19)$. Compared with the control group, S1 and ABT-737 increased the expression of LC3-II in HeLa cells (Fig. 5A and B, ${ }^{*} \mathrm{P}<0.05,{ }^{* *} \mathrm{P}<0.01$ ). Autophagy-related protein Beclin-1 is very important for the 
A
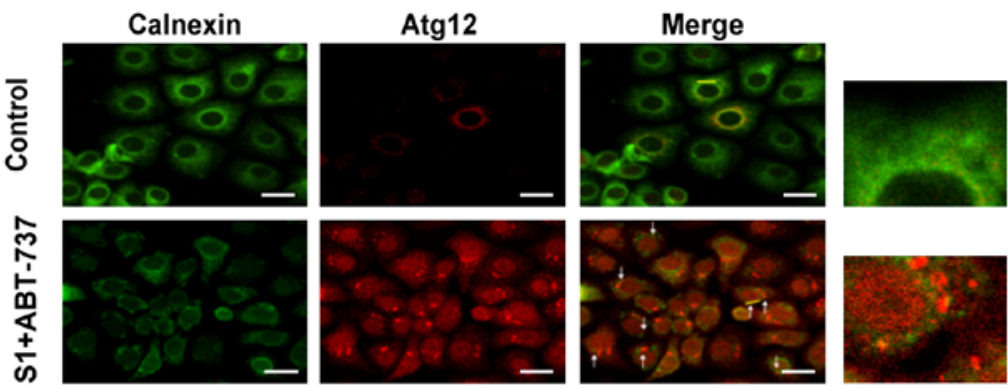

B
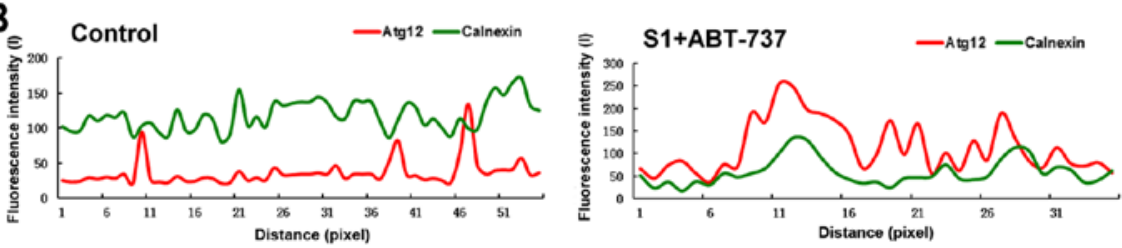

C
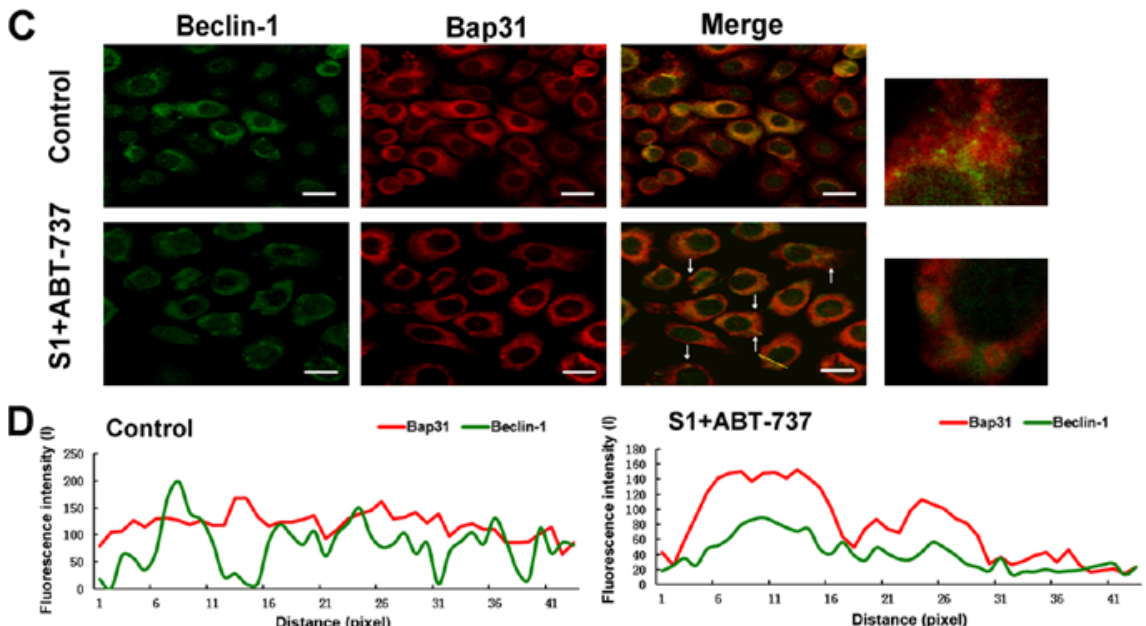

E

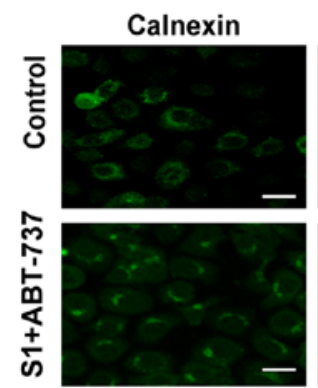

LC-3

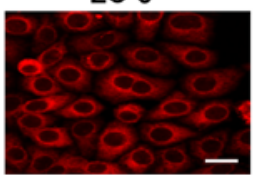

Merge
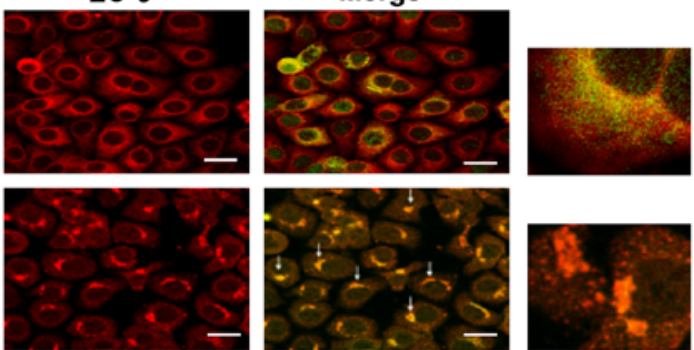

F
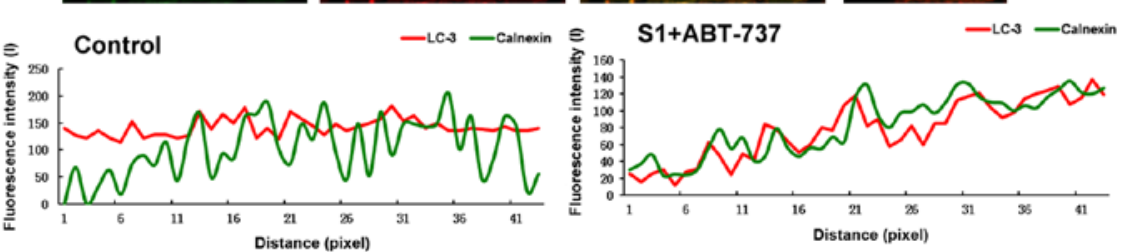

G

S1+ABT-737

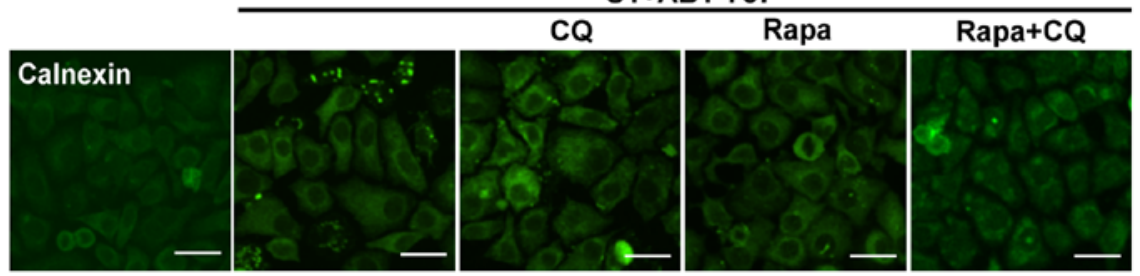

Figure 6. ER membrane remodeling is removed by autophagy. (A) Confocal microscopy of calnexin and Atg12 in the cytoplasm of HeLa cells treated with $15 \mu \mathrm{mol} / 1 \mathrm{ABT}-737$ and $8 \mu \mathrm{mol} / 1 \mathrm{~S} 1$ for $8 \mathrm{~h}$. (B) A pseudo-line scan analysis confirmed that calnexin co-localized with Atg12 induced by S1 and ABT-737 combination in HeLa cells. (C) Confocal microscopy of Bap31 and Beclin-1 in the cytoplasm of HeLa cells treated with $15 \mu \mathrm{mol} / 1 \mathrm{ABT}-737 \mathrm{and} 8 \mu \mathrm{mol} / 1 \mathrm{~S} 1$ for $8 \mathrm{~h}$. (D) A pseudo-line scan analysis confirmed that Bap31 co-localized with Beclin-1 induced by S1 and ABT-737 combination in HeLa cells. (E) Confocal microscopy of calnexin and LC3 in the cytoplasm of HeLa cells treated with $15 \mu \mathrm{mol} / 1 \mathrm{ABT}-737$ and $8 \mu \mathrm{mol} / 1 \mathrm{~S} 1 \mathrm{for} 8 \mathrm{~h}$. (F) A pseudo-line scan analysis confirmed that calnexin co-localized with LC3 induced by S1 and ABT-737 combination in HeLa cells. (G) Confocal microscopy of LC3 in the cytoplasm of HeLa cells treated with $15 \mu \mathrm{mol} / 1 \mathrm{ABT}-737$ and $8 \mu \mathrm{mol} / \mathrm{l} \mathrm{S} 1$ or combined with $10 \mathrm{mmol} / \mathrm{l} \mathrm{CQ}$, rapamycin or CQ and rapamycin in HeLa cells. 
formation of autophagosomes. The combination of S1 and ABT-737 also increased the expression of Beclin-1 after $4 \mathrm{~h}$ in HeLa cells (Fig. 5A and $\mathrm{B},{ }^{*} \mathrm{P}<0.05,{ }^{* *} \mathrm{P}<0.01$ ). In addition, visible LC3 dots in the cytoplasm were observed in HeLa cells treated with S1 combined with ABT-737 (Fig. 5C). These results indicated that autophagy does indeed occur in HeLa cells treated with S1 combined with ABT-737.

The mTOR pathway is the classical pathway that is often inhibited in autophagy $(20,21)$. Therefore, we tested the expression level of phosphorylated protein $\mathrm{p}$-mTOR involved in the mTOR pathway. However, in this study, p-mTOR remained relatively unchanged in HeLa cells treated with $\mathrm{S} 1$ combined with ABT-737 (Fig. 5A and B). Therefore, we speculated that autophagy induced by S1 combined with ABT-737 might occur through reorganization of the endoplasmic reticulum membranes.

Aggregation of the endoplasmic reticulum are removed by autophagy. The previous results showed that autophagy might be associated with decrease of endoplasmic reticulum membrane proteins. Therefore, we further investigated whether autophagy promoted the removal of membrane-reorganized endoplasmic reticulum. HeLa cells were stained with an antibody against calnexin or Bap31 to label the endoplasmic reticulum, and with antibodies against Beclin-1, Atg12, or LC-3 to label the autophagosomes. The cells analyzed by high-resolution imaging revealed extensive co-localization between autophagosomes and endoplasmic reticulum of membrane reorganized induced by the combination of $\mathrm{S} 1$ and ABT-737 in HeLa cells (Fig. 6A, C and E). A pseudo-line scan analysis confirmed that autophagosomes co-localized with endoplasmic reticulum of membrane reorganized induced by the combination of S1 and ABT-737 in HeLa cells (Fig. 6B, D and F). Rapamycin, an autophagy inducer, relieved endoplasmic reticulum membrane reorganization induced by $\mathrm{S} 1$ combined with ABT-737 in HeLa cells (Fig. 6G). In contrast, blockage of autophagy activity by $10 \mathrm{mmol} / \mathrm{l} \mathrm{CQ}$ could not delay or relieve endoplasmic reticulum membrane reorganization induced by S1 combined with ABT-737 (Fig. 6G). These results suggested that collapse/aggregation of the endoplasmic reticulum might trigger autophagy for the clearance of aggregated organelles.

\section{Discussion}

To date, numerous studies have reported that when the activities of Bcl-2 family proteins in endoplasmic reticulum were changed, endoplasmic reticulum underwent certain morphological changes, including lumen swelling, membrane fusion, reorganization, and permeabilization. Wang and colleagues used the YFP fluorescent protein to mark the endoplasmic reticulum protein PDI, and the overexpression of BAK and $\mathrm{BAX}$ in endoplasmic reticulum membrane induced the release of PDI to the cytoplasm, suggesting that BAK and BAK can increase endoplasmic reticulum membrane permeabilization (22). Hetz and colleagues found that cells treated with thapsigargin undergo endoplasmic reticulum membrane reorganization involving the massive aggregation of endoplasmic reticulum membrane, and that this reorganization was completely inhibited in cells with double knock-down
BAX and BAK, confirming that Bcl-2 family proteins could regulate the structure and dynamic changes of endoplasmic reticulum (23).

In this study, we also discovered a new kind of cell stress response that occurred after treatment with a $\mathrm{Bcl}-2$ inhibitor. The main feature of this response was the acute and recoverable bulk aggregation of the endoplasmic reticulum membrane, which preceded the changes in the expression of the endoplasmic reticulum stress response-related marker proteins GRP78 and CHOP. We discovered similar endoplasmic reticulum reorganization, including massive aggregation of endoplasmic reticulum membrane in HeLa cells with knockdown Bcl-2 and $\mathrm{Mcl}-1$, which indicated that Bcl-2 family proteins played an important role in the structure and dynamics of the endoplasmic reticulum membrane. The morphological change of the endoplasmic reticulum membrane induced by inhibition of Bcl-2 family proteins is similar to the reorganization of endoplasmic reticulum membrane induced by thapsigargin. Therefore, both of the above results suggest that Bcl-2 family proteins play a pivotal role in the structure and function of the endoplasmic reticulum.

It is worth noting that the period during which this cell stress response occurs is different from the occurrence of UPR. Several studies suggested that $\mathrm{S} 1$ and ABT-737 could up-regulate the expression of the endoplasmic reticulum stress protein Bip, and obviously increased the expression of the endoplasmic reticulum stress-related apoptotic proteins CHOP and Caspase-4, this evidence showed that S1 and ABT-737 induced apoptosis through endoplasmic reticulum stress $(8,24)$. However, we discovered that the reorganization of endoplasmic reticulum membrane preceded the changes in the expression of UPR-related proteins. We further confirmed the relationship between the time of endoplasmic reticulum membrane aggregation and the occurrence of UPR using the endoplasmic reticulum stress inhibitor TUDCA. Our results showed that TUDCA had no effect on the reorganization of endoplasmic reticulum membrane induced by $\mathrm{S} 1$ combined with ABT-737.

Autophagy is a process of eliminating partly damaged organelles, and in several studies electron microscopy showed endoplasmic reticulum and mitochondria in autophagic vacuoles $(25,26)$. Our previous work showed that Bcl-2 inhibitors could induce autophagy, and that the inhibition of autophagy can increase apoptosis. We then explored whether S1 combined with ABT-737 induced autophagy. Our results showed that the expression of the autophagy-related proteins Beclin-1, Atg12, and LC3-II increased in HeLa cells treated with S1 combined with ABT-737, in addition, we observed dot aggregation of LC-3. These results confirmed that S1 combined with ABT-737 induced autophagy in HeLa cells.

The above results indicated that the Bcl-2 inhibitors S1 and ABT-737 not only induce ER membrane reorganization but also induce autophagy, furthermore, the ER membrane reorganization occurs earlier than autophagy induced by S1 and ABT-737. When the ER membrane reorganization induced by $\mathrm{S} 1$ and ABT-737 is at high level, it will induce autophagy and autophagy will reduce the ER membrane reorganization. Therefore, the Bcl-2 inhibitors eventually reduce ER membrane reorganization possibly due to enhanced autophagy. 
Furthermore, we speculated that Bcl-2 inhibitors might cause autophagy through inducing the reorganization of the endoplasmic reticulum membrane. We were interested in whether the aggregation of endoplasmic reticulum membrane triggers autophagy, and whether this autophagy would partly eliminate endoplasmic reticulum aggregated. According to the work of Varadarajan and colleagues (5), we used the expression of the endoplasmic reticulum membrane marker proteins calnexin and Bap31 to represent the contents of endoplasmic reticulum, and we determined that S1 combined with ABT-737 obviously decreased the expression of endoplasmic reticulum membrane marker proteins, and the autophagy inhibitor 3-MA recovered the expression of endoplasmic reticulum membrane marker proteins in cells that were treated with S1 and ABT-737. These results suggested that autophagy might be related to the decrease of endoplasmic reticulum contents.

Several different factors may lead to endoplasmic reticulum fragments being degraded by sequestration into double-membrane vesicles, Hayashi-Nishino et al used electron tomography to show that early autophagic structures as a subdomain of the endoplasmic reticulum formed a cradle encircling endoplasmic reticulum in mammalian culture cells (26). Overexpression of a mutant form of Atg4B caused defects in autophagosome formation. We show a process by which aggregated portions of endoplasmic reticulum treated with S1 and ABT-737 were degraded by autophagy in HeLa cells. It is possible that autophagosomes envelop specialized areas of the endoplasmic reticulum that are aggregated. This theory is also corroborated by observations from high-resolution imaging that revealed extensive co-localization between autophagosomes and the endoplasmic reticulum of membrane reorganized induced by S1 and ABT-737 in HeLa cells. Our results showed that inhibition of autophagy by CQ could not delay or relieve S1 and ABT-737-induced endoplasmic reticulum membrane reorganization, whereas rapamycin greatly relieved endoplasmic reticulum membrane reorganization, suggesting that reorganization may trigger autophagy.

In summary, the current study showed that the S1 and ABT-737 combination triggered endoplasmic reticulum membrane reorganization in HeLa cells, and importantly autophagy played a crucial role in the clearance of damaged organelles and can be a catabolic process. Inhibition of $\mathrm{Bcl}-2$ and Mcl-1 resulted in endoplasmic reticulum membrane reorganization, and the collapsed endoplasmic reticulum then activated autophagy for clearance of aggregated organelles. Abrupt endoplasmic reticulum aggregation occurs following sequestration. Lastly, the fully formed autophagosomes acidified their contents, including aggregated organelles and then degradate them. These events provide new insight that autophagy can remove the endoplasmic reticulum membrane reorganization induced by Bcl-2 inhibitors ABT-737 and S1 and it may provide a framework for analyzing autophagy in certain diseases.

\section{Acknowledgements}

The study was supported by the National Natural Science Foundation of China (grant numbers: 81472419 and 81272876).

\section{References}

1. Kim H, Bhattacharya A and Qi L: Endoplasmic reticulum quality control in cancer: Friend or foe. Semin Cancer Biol 33: 25-33, 2015.

2. Dicks N, Gutierrez K, Michalak M, Bordignon V and Agellon LB: Endoplasmic reticulum stress, genome damage, and cancer. Front Oncol 5: 11, 2015.

3. Lin S, Sun S and Hu J: Molecular basis for sculpting the endoplasmic reticulum membrane. Int J Biochem Cell Biol 44: 1436-1443, 2012

4. English AR and Voeltz GK: Endoplasmic reticulum structure and interconnections with other organelles. Cold Spring Harb Perspect Biol 5: a013227, 2013.

5. Varadarajan S, Bampton ET, Smalley JL, Tanaka K, Caves RE, Butterworth M, Wei J, Pellecchia M, Mitcheson J, Gant TW, et al: A novel cellular stress response characterised by a rapid reorganisation of membranes of the endoplasmic reticulum. Cell Death Differ 19: 1896-1907, 2012.

6. Klee M and Pimentel-Muiños FX: Bcl-X(L) specifically activates Bak to induce swelling and restructuring of the endoplasmic reticulum. J Cell Biol 168: 723-734, 2005.

7. Nakajima K, Hirose H, Taniguchi M, Kurashina H, Arasaki K, Nagahama M, Tani K, Yamamoto A and Tagaya M: Involvement of BNIP1 in apoptosis and endoplasmic reticulum membrane fusion. EMBO J 23: 3216-3226, 2004.

8. Zhong JT, Xu Y, Yi HW, Su J, Yu HM, Xiang XY, Li XN, Zhang ZC and Sun LK: The BH3 mimetic S1 induces autophagy through ER stress and disruption of Bcl-2/Beclin 1 interaction in human glioma U251 cells. Cancer Lett 323: 180-187, 2012.

9. Malik SA, Shen S, Mariño G, BenYounès A, Maiuri MC and Kroemer G: BH3 mimetics reveal the network properties of autophagy-regulatory signaling cascades. Autophagy 7: 914-916, 2011.

10. Rubinsztein DC, Gestwicki JE, Murphy LO and Klionsky DJ: Potential therapeutic applications of autophagy. Nat Rev Drug Discov 6: 304-312, 2007.

11. Quinsay MN, Thomas RL, Lee Y and Gustafsson AB: Bnip3mediated mitochondrial autophagy is independent of the mitochondrial permeability transition pore. Autophagy 6: $855-862,2010$

12. Naydenov NG, Harris G, Morales V and Ivanov AI: Loss of a membrane trafficking protein $\alpha$ SNAP induces non-canonical autophagy in human epithelia. Cell Cycle 11: 4613-4625, 2012.

13. Zhang B, Xie C, Zhong J, Chen H, Zhang H and Wang X: A549 cell proliferation inhibited by RNAi mediated silencing of the Nrf2 gene. Biomed Mater Eng 24: 3905-3916, 2014.

14. Heath-Engel HM, Chang NC and Shore GC: The endoplasmic reticulum in apoptosis and autophagy: Role of the BCL-2 protein family. Oncogene 27: 6419-6433, 2008.

15. Long MJ, Gollapalli DR and Hedstrom L: Inhibitor mediated protein degradation. Chem Biol 19: 629-637, 2012.

16. Maejima Y, Kyoi S, Zhai P, Liu T, Li H, Ivessa A, Sciarretta S, Del Re DP, Zablocki DK, Hsu CP, et al: Mst1 inhibits autophagy by promoting the interaction between Beclin1 and Bcl-2. Nat Med 19: 1478-1488, 2013.

17. Hanna RA, Quinsay MN, Orogo AM, Giang K, Rikka S and Gustafsson AB: Microtubule-associated protein 1 light chain 3 (LC3) interacts with Bnip3 protein to selectively remove endoplasmic reticulum and mitochondria via autophagy. J Biol Chem 287: 19094-19104, 2012.

18. Tooze SA, Jefferies HB, Kalie E, Longatti A, McAlpine FE, McKnight NC, Orsi A, Polson HE, Razi M, Robinson DJ, et al: Trafficking and signaling in mammalian autophagy. IUBMB Life 62: 503-508, 2010.

19. Yang Z and Klionsky DJ: Mammalian autophagy: Core molecular machinery and signaling regulation. Curr Opin Cell Biol 22: 124-131, 2010.

20. Paglin S, Lee NY, Nakar C, Fitzgerald M, Plotkin J, Deuel B, Hackett N, McMahill M, Sphicas E, Lampen N, et al: Rapamycinsensitive pathway regulates mitochondrial membrane potential, autophagy, and survival in irradiated MCF-7 cells. Cancer Res 65: 11061-11070, 2005.

21. Takeuchi H, Kondo Y, Fujiwara K, Kanzawa T, Aoki H, Mills GB and Kondo S: Synergistic augmentation of rapamycin-induced autophagy in malignant glioma cells by phosphatidylinositol 3-kinase/protein kinase B inhibitors. Cancer Res 65: 3336-3346, 2005. 
22. Wang $\mathrm{X}$, Olberding KE, White $\mathrm{C}$ and $\mathrm{Li} \mathrm{C}$ : $\mathrm{Bcl}-2$ proteins regulate ER membrane permeability to luminal proteins during ER stress-induced apoptosis. Cell Death Differ 18: 38-47, 2011.

23. Hetz C, Bernasconi P, Fisher J, Lee AH, Bassik MC, Antonsson B Brandt GS, Iwakoshi NN, Schinzel A, Glimcher LH, et al: Proapoptotic BAX and BAK modulate the unfolded protein response by a direct interaction with IRE1alpha. Science 312: 572-576, 2006

24. Liu N, Xu Y, Sun JT, Su J, Xiang XY, Yi HW, Zhang ZC and Sun LK: The BH3 mimetic S1 induces endoplasmic reticulum stress-associated apoptosis in cisplatin-resistant human ovarian cancer cells although it activates autophagy. Oncol Rep 30: 2677-2684, 2013.
25. Schuck S, Gallagher CM and Walter P: ER-phagy mediates selective degradation of endoplasmic reticulum independently of the core autophagy machinery. J Cell Sci 127: 4078-4088, 2014.

26. Hayashi-Nishino M, Fujita N, Noda T, Yamaguchi A, Yoshimori T and Yamamoto A: A subdomain of the endoplasmic reticulum forms a cradle for autophagosome formation. Nat Cell Biol 11: 1433-1437, 2009. 